\title{
Fluorescence lifetime imaging of red yeast Cystofilobasidium capitatum during growth
}

\author{
Martin Vanek ${ }^{1}$, Filip Mravec ${ }^{1}$, Martin Szotkowski ${ }^{1}$, Dana Byrtusova ${ }^{1}$, Andrea Haronikova ${ }^{1}$, Milan Certik ${ }^{2}$, \\ Volha Shapaval $^{3}$ and Ivana Marova ${ }^{1 *}$
}

\begin{abstract}
Red yeast Cystofilobasidium capitatum autofluorescence was studied by means of confocal laser scanning microscopy (CLSM) to reveal distribution of carotenoids inside the cells. Yeasts were cultivated in $2 \mathrm{~L}$ fermentor on glucose medium at permanent light exposure and aeration. Samples were collected at different times for CLSM, gravimetric determination of biomass and HPLC determination of pigments. To compare FLIM (Fluorescence Lifetime Imaging Microscopy) images and coupled data (obtained by CLSM) with model systems, FLIM analysis was performed on micelles of SDS:ergosterol and SDS:coenzyme Q with different content of ergosterol and coenzyme Q, respectively, and with constant addition of beta-carotene. Liposomes lecithin:ergosterol:beta-carotene were investigated too. Two different intracellular forms of carotenoids were observed during most of cultivations, with third form appeared at the beginning of stationary phase. Observed behavior is probably due to formation of some kind of carotenoid protective system in membranes of different compartments of yeast cell, especially cytoplasmic membrane.
\end{abstract}

Keywords: autofluorescence, carotenoids, Cystofilobasidium capitatum, fluorescence lifetime imaging, red yeasts

${ }^{1}$ Materials Research Centre, Faculty of Chemistry BUT, Brno, Czech Republic,

${ }^{2}$ Faculty of Chemical and Food Technology, Slovak University of Technology, Bratislava, Slovak Republic

${ }^{3}$ Norwegian University of Life Sciences, As, Norway

${ }^{*}$ Corresponding author: I. Marova

E-mail: marova@fch.vut.cz

DOI: 10.2478/ebtj-2018-0015
(C) 2018 Authors. This work was licensed under the Creative Commons AttributionNonCommercial-NoDerivs 3.0 License.

\section{Introduction}

Carotenoids are one of the most abundant natural pigment and they have great potential for wide industrial utilization in food and feed industry, cosmetics, pharmacy and even electronics $(1,2)$. Carotenoids can be obtained by means of biotechnological production, besides using so-called red yeasts strains while utilizing waste substrates. For higher production of carotenoids from red yeasts, application of some type of external stress $(3,4)$ was described. Furthermore, red yeasts also accumulate lipids which broaden their industrial potential as a source of biodiesel from waste materials, e.g. lignocellulose, or as a source of lipid enhanced biomass for feed industry.

Accumulation of carotenoids inside cells mean significant enlargement of cells autofluorescence due to intrinsic fluorescence of these pigments after blue light excitation, even though the quantum yield of fluorescence is very low $-1.7 \cdot 10^{-4}$ for $\beta$-carotene (5). Autofluorescence is mostly considered as an obstacle, but this time due to specific distribution of carotenoids inside the cells (in lipophilic structures) can be grasped as a tool for investigation and visualisation of carotenoids and lipids formation and storage inside the cells. For such an investigation high resolution techniques like laser scanning confocal microscopy (LSCM) should have to be used.

Comparison of model systems (liposomes, micelles) with real natural structures of cells is rather unique in this field. Carotenoid-lipid interactions were studied only in the context of delivery systems (6), and fluorescence is also frequently used for such studies, but not as a carotenoid lifetime measurement like in the presented work. 
Because of complex fluorescent environment inside the cells (e.g. NAD/NADH, FAD, cytochromes) excited by blue light and with emission in green region of spectrum, simple intensity imaging is not appropriate for gaining such information about carotenoids and lipid metabolism. For resolution between every single fluorescent species we used fluorescence lifetime imaging microscopy (FLIM), which profits from combination of state-of-the-art time correlated single photon counting electronics coupled with laser scanning confocal microscope (MicroTime 200, PicoQuant GmbH, Germany).

There was done a lot of work on Saccharomyces cerevisiae yeast using fluorescence microscopy. Valuable review of LSCM applied on yeasts $S$. cerevisiae is summarized in (7). This review is dealing with staining both glycogen and neutral lipids as a storage material, staining inner membranes (like vacuolar membrane), nucleic acids and bud scars. Also green fluorescent protein tagging was mentioned in this review as a tool with high potential to reveal metabolic relationships. In the focus of our work is lipid metabolism, where LSCM is also used as imaging technique when studying $S$. cerevisiae as a model organism. Lipids are accumulated in all eukaryotic cells as lipid bodies (LB), which are not a simple warehouse, but rather one of the principal compartments with function including protein degradation, temporal protein storage and also signaling (8 10).

One reason for study such a complex system with LSCM was proposed as statement (11), that morphological alteration of LB is affected by processes maintaining neutral lipid and phospholipid homeostasis. Some of the red yeasts were found accumulating of huge amounts of lipids in the form of big, „supersized" LB (more than $1 \mu \mathrm{m}$ in diameter). Such big droplets were studied in S. cerevisiae using fluorescence microscopy and coalescence of droplets was found as a mechanism of origin, mediated probably by high intracellular amounts of phosphatidic acid and phosphatidylethanolamine (12). Furthermore, to gain more complexity, another mechanism of triacylglycerol biosynthesis (different from endoplasmic reticulum mechanism, leading to LB) was found in Rhodotorula glutinis red yeast cytosolic soluble diacyltransferase, which contribution to LB formation is unrevealed (13).

Although there is plenty of fluorescence microscopy work on Saccharomyces cerevisiae yeast, the red yeasts remain tabula rasa. So, such a measurement is unique on red yeasts - based on our expert knowledge, there is only one similar work (14). Unfortunately, that work was addressed to quantification of carotenoid content by means of intensity imaging; the intriguing nature of lipid and carotenoid metabolism was only proposed to be assessed by LSCM. However, knowledge about lipids and carotenoids formation and storage during culture development is of high importance for biotechnological applications - understanding these processes means better possibilities to alter fermentations in favor to higher yields and good economical balance.

\section{Materials and Methods \\ Cultivation of microorganisms}

Carotenogenic yeast strain Cysofilobasidium capitatum CCY 10-1-2 was obtained from Culture Collection of Yeasts (CCY; Institute of Chemistry, Slovak Academy of Sciences, Bratislava, Slovak Republic) and preserved on malt agar, stored at $4{ }^{\circ} \mathrm{C}$ in darkness.

Cystofilobasidium capitatum (CCY-10-1-2) was cultivated in 21 bioreactor Biostat ${ }^{\oplus} \mathrm{B}$ plus (Sartorius) on glucose medium (60 $\mathrm{g} / \mathrm{l})$. Two-step inoculation was performed in Erlenmeyer flasks in the optimal inoculation medium (in g/l: glucose 40.0, $\left(\mathrm{NH}_{4}\right)$ $\left.{ }_{2} \mathrm{SO}_{4}\right) 5.0, \mathrm{KH}_{2} \mathrm{PO}_{4} 5, \mathrm{MgSO}_{4} \cdot 7 \mathrm{H}_{2} \mathrm{O} 0.696$, yeast extract 7.0$)$. The first inoculum $(50 \mathrm{ml})$ was cultivated for $24 \mathrm{~h}$ at $28^{\circ} \mathrm{C}$ under continuous lighting and shaking. Inoculum I was then transferred into $0.25 \mathrm{l}$ of fresh inoculum II, which was grown under the same conditions as inoculum I. After $24 \mathrm{~h}$, inoculum II was transferred into a fermentor containing $1.5 \mathrm{l}$ of sterile production medium (in g/l: glucose 60.0, $\left.\left(\mathrm{NH}_{4}\right)_{2} \mathrm{SO}_{4}\right)$ 5.0, $\mathrm{KH}_{2} \mathrm{PO}_{4}$ 5.0, $\mathrm{MgSO}_{4} \cdot 7 \mathrm{H}_{2} \mathrm{O} 0.696$, yeast extract 7.0$)$. Batch cultivation in a fermentor was carried out at $28^{\circ} \mathrm{C}, \mathrm{pH} 5.5$, constant aeration (2 $1 / \mathrm{min}$ ) and under permanent light exposure (11 W fluorescent bulb on the outer side of the fermentor) and stirring (DOT set to $30 \%)$. As an initial screening, samples were collected after $6,22,30,46$ and 52 hours of cultivation for FLIM, gravimetric determination of biomass and RP-HPLC/PDA determination of pigments.

For comparison, cultivation on agar plate was performed on very microscope stage, thus providing the possibility to measure cells with higher frequency. Flow cell chamber FCS2 (Bioptechs) was used for cultivation on agar. Constitution of agar medium was the same as inoculation medium (see above), but

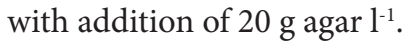

\section{Preparation of model systems}

To compare FLIM images and coupled data with model systems, FLIM analysis was performed on micelles of SDS:ergosterol and SDS:coenzyme Q with different content of ergosterol and coenzyme $\mathrm{Q}$, respectively, and with constant addition of $\beta$-carotene. Liposomes lecithin:ergosterol: $\beta$-carotene were investigated too. Liposomes were prepared by thin layer evaporation (TLE). Ergosterol, coenzyme Q, lecithin and $\beta$-carotene were purchased from Sigma-Aldrich.

Micelles were prepared by dissolution of components in chloroform, mixing them up in required ratio and then chloroform was evaporated. Thereafter, dry thin layer was hydrated with distilled water on laboratory ultra-sonicator (Powersonic PS 02000 ) for $15 \mathrm{~min}$ at power of $40 \mathrm{~W}$. Concentration of $\beta$-carotene was held constant at $0.1 \mathrm{mM}$ and concentration of SDS was set to $12 \mathrm{mM}$ for all solutions. Modification of these micelles was done by addition of coenzyme Q with concentration ranging from 0 to $1.25 \mathrm{mM}$, or by addition of ergosterol with concentration ranging from 0 to $1.25 \mathrm{mM}$.

Liposomes were prepared by TLE of primary mixture of above mentioned components dissolved in chloroform. Then the hydration was done the same manner like mentioned above. 
Table 1. Carotenoid fluorescence lifetimes in lipid clusters in different structures - micelles, liposomes and cells (lipid bodies and membranes)

\begin{tabular}{|c|c|c|c|c|c|c|c|}
\hline & \multicolumn{2}{|c|}{ Micelles with ergosterol } & \multicolumn{2}{|c|}{ Micelles with CoQ } & \multicolumn{2}{c|}{$\begin{array}{c}\text { Lultivation on } \\
\text { agar }\end{array}$} \\
\hline & $\tau(\mathrm{ns})$ & $\begin{array}{c}\text { Relative abun- } \\
\text { dance (\%) }\end{array}$ & $\tau(\mathrm{ns})$ & $\begin{array}{c}\text { Relative } \\
\text { abundance (\%) }\end{array}$ & $\tau(\mathrm{ns})$ & $\begin{array}{c}\text { Relative } \\
\text { abundance (\%) }\end{array}$ \\
\hline$\tau_{1}$ & 0.013 & 85.8 & 0.013 & 79 & 0.033 & 73.14 & $0.04^{\mathrm{b}}$ \\
\hline$\tau_{2}$ & $0.034^{\mathrm{a}}$ & 14.1 & $0.028^{\mathrm{a}}$ & 20.95 & 0.297 & 10.42 & 0.303 \\
\hline$\tau_{3}$ & 0.356 & 0.07 & 0.464 & 0.02 & 1.112 & 7.07 & 0.7 \\
\hline$\tau_{4}$ & 3.2 & 0.04 & 3.145 & 0.03 & 3.94 & 9.36 & 3.5 \\
\hline
\end{tabular}

a lifetimes with negative amplitudes, ${ }^{b}$ lifetimes with negative amplitudes occur only in cells in stationary phase

Concentration of $\beta$-carotene was held constant at $0.6 \mu \mathrm{M}$ and concentration of lecithin was set to $1.2 \mathrm{mM}$ for all solutions. Modification of liposomes was done by addition of coenzyme $\mathrm{Q}$ with concentration ranging from 0.012 to $0.5 \mathrm{mM}$.

\section{Fluorescence Lifetime Imaging Microscopy (FLIM)}

FLIM was performed on MicroTime 200 machine (PicoQuant $\mathrm{GmbH}$, Germany), with $467 \mathrm{~nm}$ laser diode head for excitation (80 MHz pulse rate), 520/35 nm emission filter (Semrock, USA), using inverted water immersion objective Olympus UPLSAPO 60XW (60x mag, NA 1.2).

For data processing we used SymPhoTime software supplied by manufacturer of the machine. Data were analyzed pixel by pixel to obtain each fluorescent species lifetime, amplitude and intensity.

The model systems samples and samples from fermentor were measured directly on the cover glass placed on the objective. Semi-continuous monitoring of culture development was performed on agar in flow cell chamber FCS2 (Bioptechs, USA). This equipment is appropriate for long course measurement right on the microscope stage while protecting culture from negative effects of surrounding environment.

\section{Carotenoids extraction and HPLC analysis}

During culture growth in fermentor $50 \mathrm{ml}$ samples were collected for carotenoid extraction and analysis. Samples were then centrifuged at $4500 \mathrm{RCF}$ for $10 \mathrm{~min}$ (Sigma 3-15, Sigma Laborzentrifugen $\mathrm{GmbH}$, Germany), washed in distilled water and centrifuged again. The pellet was then re-suspended in acetone and mechanically disrupted by pestle and mortar. After saponificantion by $20 \%$ ethanolic solution of $\mathrm{KOH}$ at $90^{\circ} \mathrm{C}$ extraction by diethylether was performed and the extract was dried on rotary evaporator. Dry extract was dissolved in 1-2 ml of UV-VIS grade ethanol.

Samples were then filtered using $0.45 \mu \mathrm{m}$ PTFE filters and $10 \mu \mathrm{l}$ of each sample was injected onto column Kinetex C18 $5 \mu \mathrm{m}, 150 \times 4.6 \mathrm{~mm}$ (Phenomenex, USA) with guard column $5 \mu \mathrm{m}, 30 \times 4.6 \mathrm{~mm}$, both equilibrated to $45^{\circ} \mathrm{C}$ with methanol as elution solvent (flowrate $1 \mathrm{ml} / \mathrm{min}$ ), on Thermo Finnigan
Surveyor HPLC system. Xcalibur software was used for chromatography data analysis. Carotenoids content was evaluated according to previous study (15), i.e. individual carotenoids were identified using RP-HPLC/PDA and their content was evaluated using calibration curve constructed with $\beta$-carotene standard solutions with concentration ranging 10-100 $\mu \mathrm{g} / \mathrm{mL}$. B-carotene standard was purchased from Sigma-Aldrich.

\section{Results}

\section{Analysis of carotenoids fluorescence in model systems}

Investigation of model systems showed the presence of four different lifetimes (see Table 1 ), one of them ( $\approx 40$ ps) with negative value of amplitude due to excited state kinetics of slowly rotating molecules in environment with high viscosity. Considering the nature of model systems - liposomes and micelles - it should be stated that rotation and diffusion is also constrained in such systems. No significant variance of lifetimes values with concentration of ergosterol or coenzyme Q (CoQ) was observed (data not shown), with exception of the longest lifetime (see Fig. 1). It should be mentioned that this lifetime was significantly longer in liposomes than in micelles and occurs more frequently in liposomes ( $9 \%$ vs $0.03 \%$ - see Table 1 ). This indicates substantially higher level of excited state stabilization in liposomes.

Although lifetimes generally do not change with varying concentration of trigs (ergosterol or CoQ) in model lipidic structures, relative abundances (derived as relative amplitudes) changed moderately (see Fig. 2 and Fig. 3 for comparison of ergosterol influence in micelles and liposomes, respectively). Both in micelles and liposomes the shortest lifetime was overwhelmingly abundant (around 80\%). In micelles, significantly abundant was also negative-amplitude lifetime while the other lifetimes were rare. In liposomes there was found more equality in contribution to overall fluorescence between all other lifetimes than the shortest one.

\section{Evaluation of FLIM data for cultivation on agar}

Based on data evaluation, in red yeast cells only two carotenoid lifetimes were significant - the longest one, assigned as Carot- 


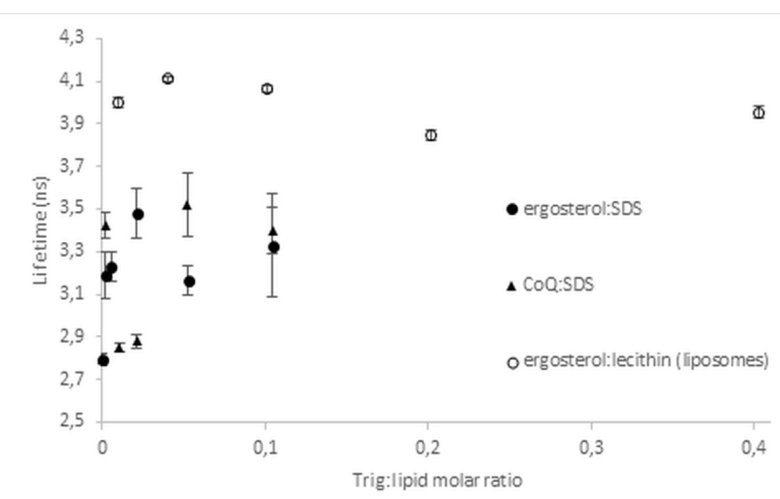

Figure 1. Carotenoids I lifetime of $\beta$-carotene $( \pm S D)$ at different trigs-to-lipid ratio in model systems.

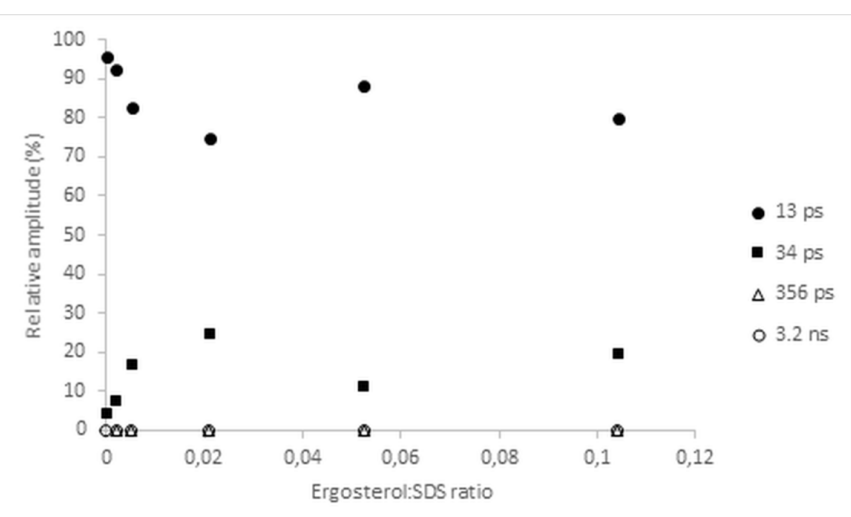

Figure 2. Relative abundances of different $\beta$-carotene lifetimes in ergosterol:SDS micelles.

Note: Relative abundances were derived as relative amplitudes. Abundances of 3.2 ns and 356 ns lifetimes are very low and, thus, they are overlapping in the graph.

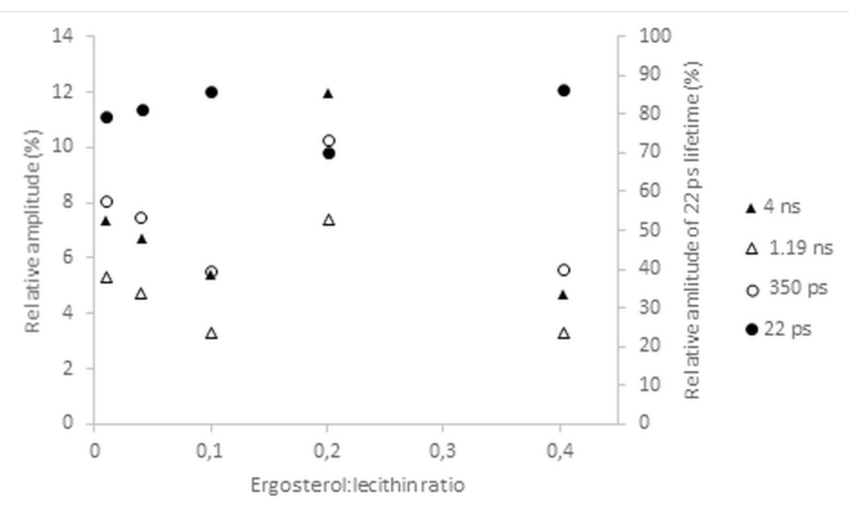

Figure 3. Relative abundances of different $\beta$-carotene lifetimes in ergosterol:lecithin liposomes. Relative abundances were derived as relative amplitudes.

enoids I, and the shortest one, assigned as Carotenoids II. The third lifetime with negative values of amplitude does occur only in specific phases of culture development. Simultaneously with carotenoid fluorescence, the $\mathrm{NADP}(\mathrm{H})$ autofluorescence (16) was observed when measured in green region of spectra. Variable relative intensity of this $\operatorname{NADP}(\mathrm{H})$ fluorescence ranging up to $25 \%$ (see Fig. 4) was observed.

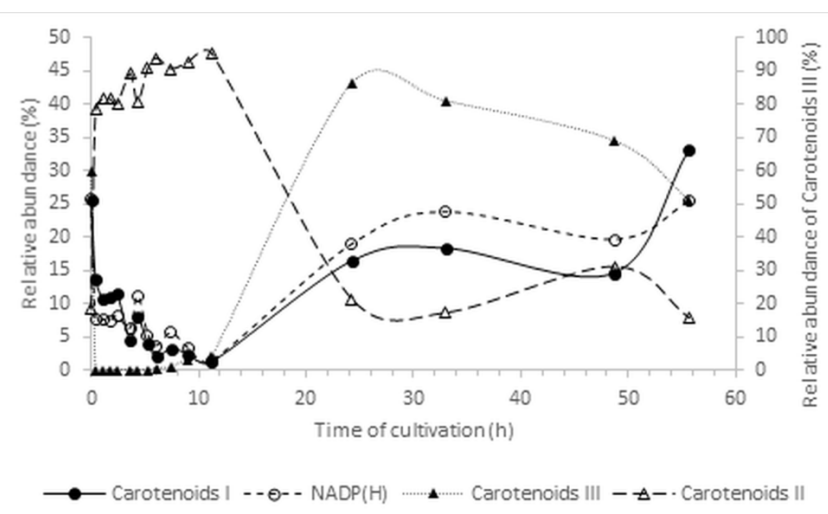

Figure 4. Relative abundance of lifetimes during growth on agar.

Note: Lifetimes: Carotenoids I ( $\approx 3.5 \mathrm{~ns}), \mathrm{NADP}(\mathrm{H})(\approx 0.6 \mathrm{~ns})$, carotenoids II ( $\approx 0.03 \mathrm{~ns})$, carotenoids III ( $\approx 0.3 \mathrm{~ns})$.

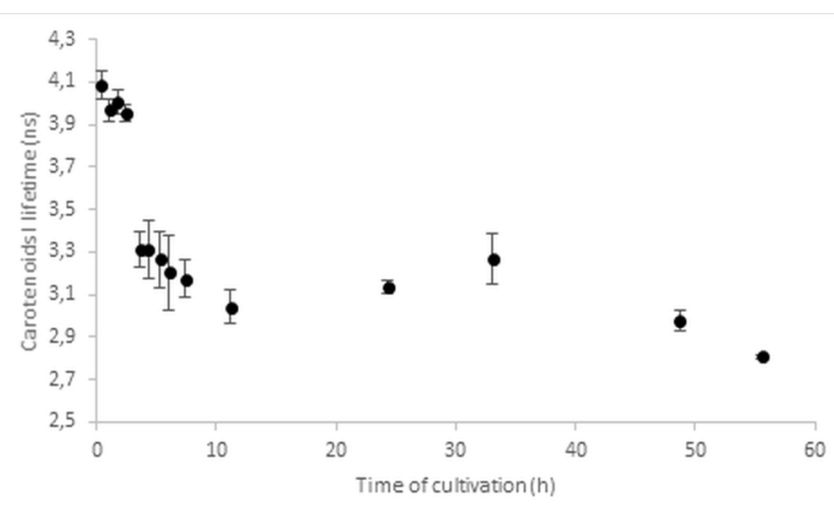

Figure 5. Carotenoids I lifetime value during growth on agar. Note: Each point is an average from 2-3 groups of cells measured at that time.

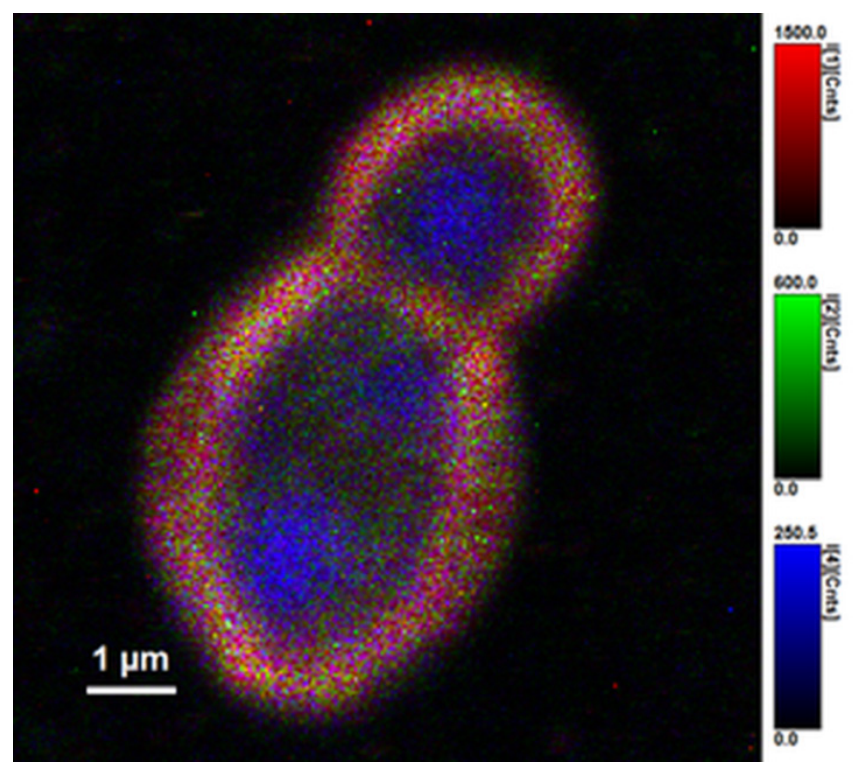

Figure 6. Cystofilobasidium capitatum cell half an hour after inoculation to solid media in flow cell.

Note: False-colour representation used: red - Carotenoids I, green - NADP(H), blue - Carotenoids II. Cells were inoculated from late stationary phase when preserved on malt agar media at $4{ }^{\circ} \mathrm{C}$. Cytosolic membrane appeared to be stacked with carotenoids as well as lipid bodies inside the cell. 

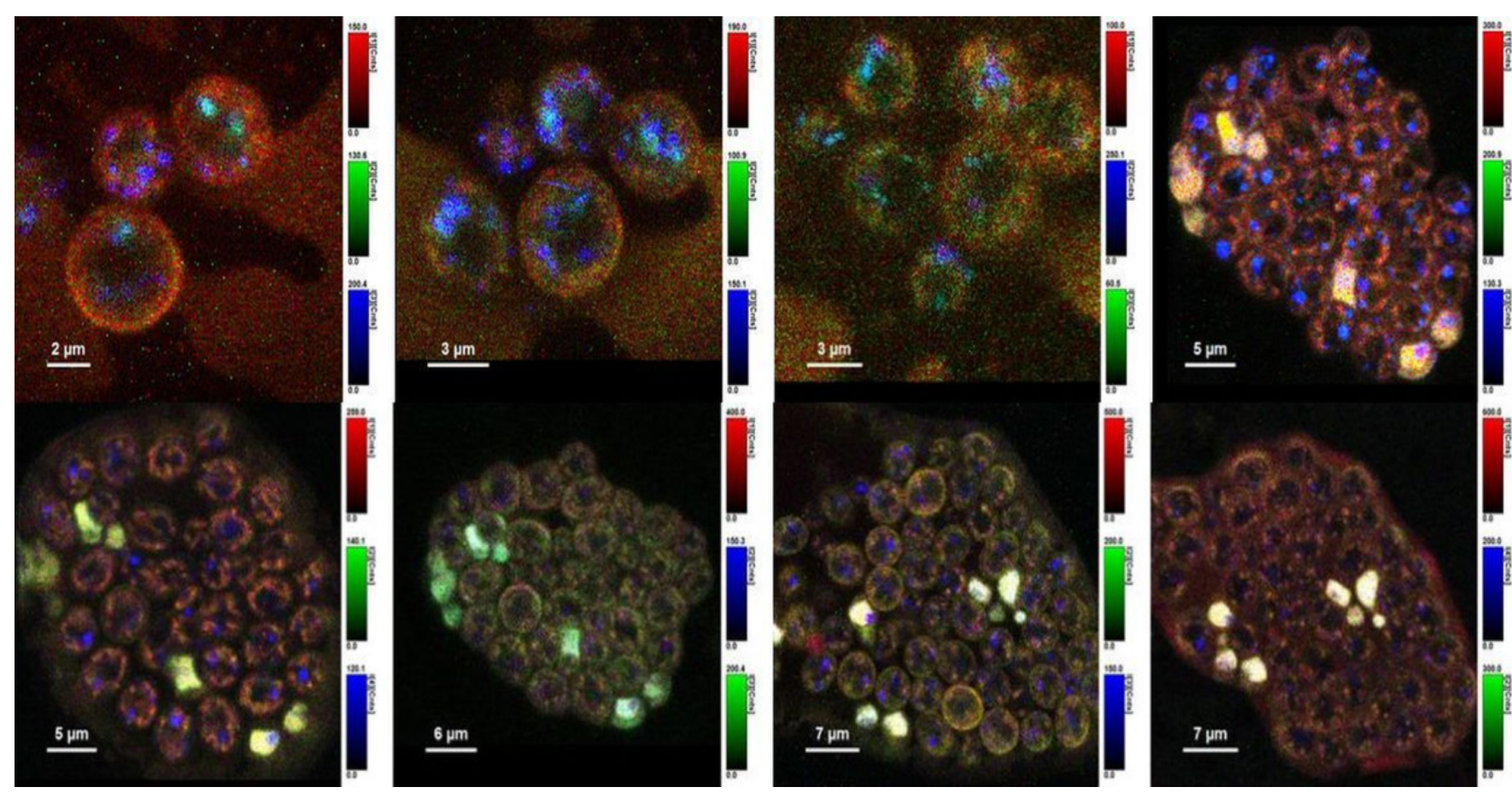

Figure 7. Cystofilobasidium capitatum cells during growth on agar.

Note: False-colour representation used: red - Carotenoids I, green - NADP(H), blue - Carotenoids II. Top line from the left: approximately 4, 5.5, 7.5 and 11.5 hours after inoculation; bottom line from the left: 24, 33, 49 and 56 hours after inoculation. At 4, 5.5 and 7.5 hours, when in exponential phase, it can be seen that Carotenoids I (membrane form) is decreasing.

Carotenoids I lifetimes show very distinct time pattern. When focused on the start of cultivation, a sudden drop was observed when cells move from lag to exponential phase. This drop was observed both in lifetimes and relative abundances (Fig. 4 and Fig. 5). This drop can be seen as disappearing of Carotenoids I, the membrane form (Fig. 6 and Fig. 7). A dropped value of Carotenoids I remain even if the culture move from exponential to stationary phase, up to $56^{\text {th }}$ hour, when the observation was stopped.

When considering relative abundance (Fig. 4), Carotenoids II, which occurs in lipid bodies inside cells, increased mildly with entering to exponential phase (from $40 \%$ to $45 \%$ ), where remain constant. This is a bit contradictory to our belief that storage lipids should be consumed during exponential phase as a source of energy and carbon for biosynthesis. When culture moves to stationary phase, this form typical for storage lipid bodies rapidly drops in cell content but another form of carotenoids, assigned as Carotenoids III (lifetime $\approx 0.3 \mathrm{ns,} \mathrm{in} \mathrm{range}$ $0.2-0.4$ ns respectively), rapidly grows. When comparing with model systems, we hypothesized that this form exists in cells as a micelles or transport vesicles of lipids containing bunch of carotenoids, and these micelles/vesicles are metabolically active forms of cell lipids. Furthermore, $\operatorname{NADP}(\mathrm{H})$ fluorescence and Carotenoids I fluorescence also increased, supporting the idea of synthesizing and transporting carotenoids into cytoplasmic membrane or other organelle membranes, where it serves as a protective system.

\section{Evaluation of FLIM and HPLC data for cultivation in fermentor}

Simple overview of culture development monitored by FLIM is in Fig. 8 as a screening for method application. Lifetimes of Carotenoids I during cultivation were roughly comparable with that of agar cultivation. Only Carotenoids I, Carotenoids II and NADP(H) fluorescence was observed, not the micellar form - Carotenoids III. Relative abundances (see Fig. 9) shows massive change of Carotenoids I: Carotenoids II ratio during start of stationary phase, right the other way as it was when cultivating on solid medium. It should be hypothesized that during deep stationary phase the ratio will be shifted in the other way to protect cells from harm. Longer cultivation and sampling for examination this hypothesis should be an object of further research.

Summed fluorescence intensities of both observed forms of carotenoids (data not shown) show similarity with total carotenoids content determined by HPLC and suggests kind of quadratic dependence on cultivation time, while beta-carotene content enlarge in a linear way.

\section{Discussion}

In the first part of present work some model systems were proposed. Observed stabilization of carotenoid excited state in liposomes is probably due to preferential orientation of carotenoid molecules across the membrane. So, other carotenoid molecules in surrounding have their $\pi$ molecular orbitals aligned with those in excited molecule and can stabilize it by means of resonance. This point of view suggests idea of highly 


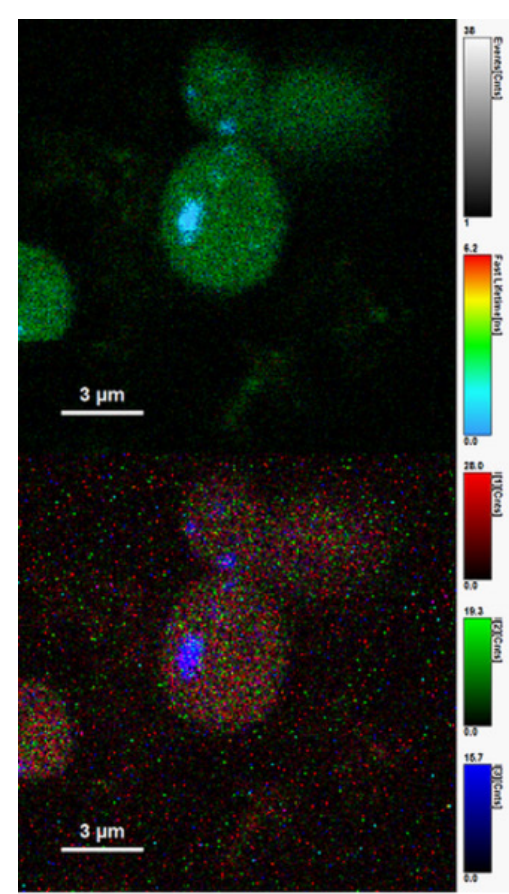

exponential phase

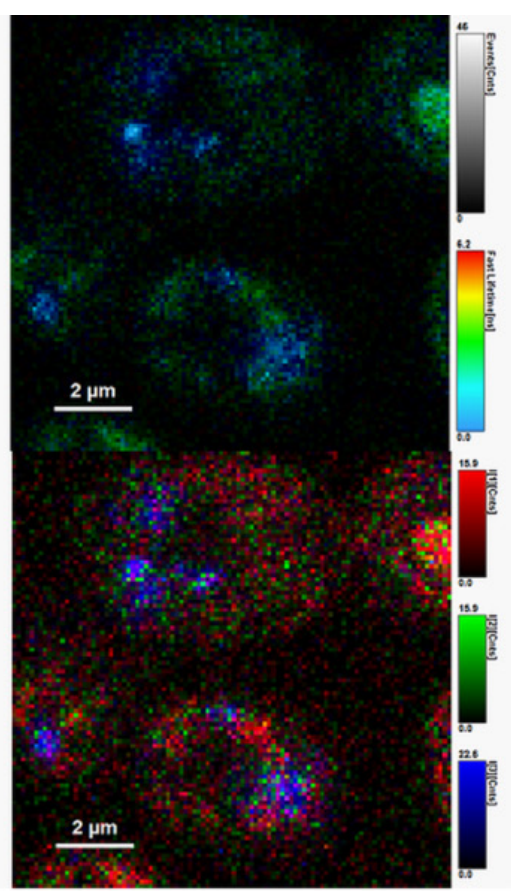

early stationary phase

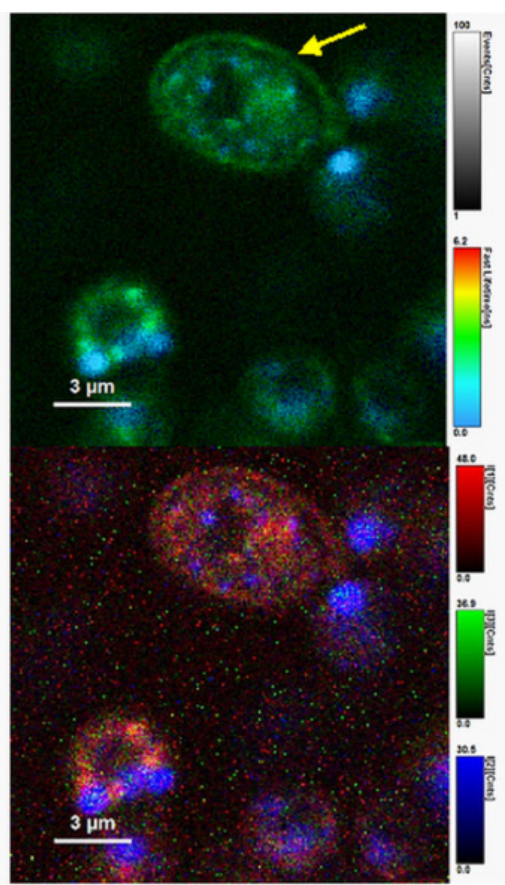

stationary phase

Figure 8. Cystofilobasidium capitatum cells during fermentation in 21 laboratory fermentor.

Note: In liquid media the cells show different patterns during time. In the upper part row intensity based preliminary lifetime images are illustrated, while row FLIM images with falsecolour representation (as mentioned above) are at the bottom. In exponential phase Carotenoid I lifetime seems to be disappeared, while in early stationary phase this membrane form is concentrated in endoplasmic reticle. In the stationary phase this form is present both in endoplasmic reticle and cytosolic membrane (yellow arrow).

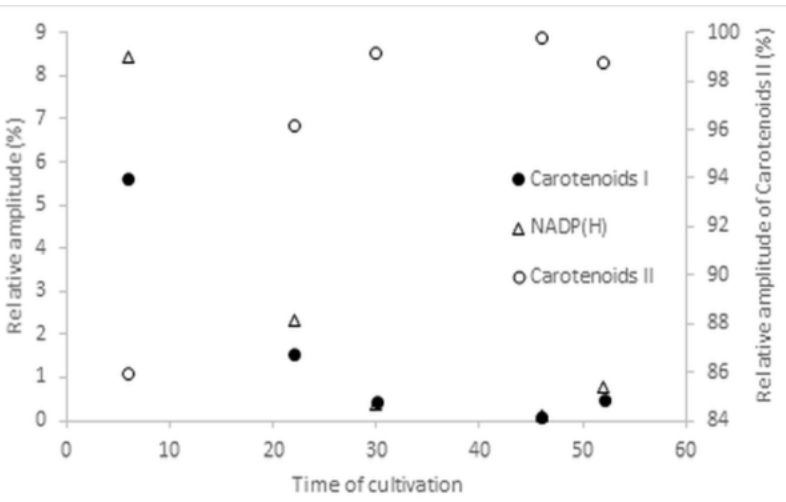

Figure 9. Relative abundance changes during fermentation.

efficient carotenoid protection system in (yeast) membranes. Meanwhile, distributions of lifetimes found in micelles point out randomly oriented arrangement of carotenoid molecules in micelles (carotenoid:SDS ratio 1:120, when SDS aggregation number should be considered $\approx 60$ ).

Observed disappearance of "membrane form" Carotenoid I when entering to exponential phase of growth can be due to simple dilution of carotenoids when synthesizing new membranes during cell division, or due to consumption of carotenoids as an energy and carbon source (17), similarly to observation of germinating aplanospores of Haematococcus pluvialis. Such possible consumption of carotenoids in yeasts should be further investigated. Importantly, a dropped value of Carotenoids I remain even if the culture move from exponential to stationary phase, up to $56^{\text {th }}$ hour, when the observation was stopped. This fact points out a very distinct structure of membrane carotenoids in deep stationary phase during long term storage of culture on solid media, which seems like to be intensively stabilized in sense of excited state stabilization (see above).

When culture on agar moves to stationary phase, carotenoid form typical for storage lipid bodies rapidly drops in content, but different form of carotenoids assigned as Carotenoids III (lifetime $\approx 0.3 \mathrm{~ns}$, in range $0.2-0.4 \mathrm{~ns}$ respectively) rapidly grows. This lifetime corresponds to one of lifetimes found in micelles and we hypothesized that observed arise of Carotenoids III means formation of micelles or transport vesicles of lipids containing bunch of carotenoids partially oriented. These micelles/vesicles are transported to cytoplasmic. This idea is supported with increasing $\operatorname{NADP}(\mathrm{H})$ and Carotenoids I fluorescence. Observed behavior is probably due to formation of some kind of carotenoid protective system in membranes of different compartments of yeast cell, especially cytoplasmic membrane.

Both on solid media and in liquid media (fermentor) relatively high levels of $\mathrm{NADP}(\mathrm{H})$ fluorescence were detected. Variable relative intensity of this $\operatorname{NADP}(\mathrm{H})$ fluorescence was ranging up to $20 \%$ (see Fig. 5). Thus, it should be noted that flow cytometric determination of carotenoids (18-20) is probably always burdened with significant error. However, it was proven as eligible (14). 
Discovered difference between carotenoids accumulation when cultivating on solid or liquid media, respectively, are very inspiring and required more detailed research. When considered better oxygenation and substrate availability in fermentor, it is obvious to think that cells are less stressed so they can accumulate more lipids and carotenoids in storage lipid bodies instead of creating protective system in plasmatic membrane.

\section{Conclusions}

Red yeast Cystofilobasidium capitatum autofluorescence was studied by means of confocal laser scanning microscopy (CLSM) to reveal distribution of carotenoids inside the cells. Yeasts were cultivated in solid and liquid glucose medium at permanent light exposure and aeration. To compare FLIM (Fluorescence Lifetime Imaging Microscopy) images and coupled data (obtained by CLSM) with model systems, FLIM analysis was performed on micelles of SDS:ergosterol and SDS:coenzyme Q. Two different intracellular forms of carotenoids were observed during most of cultivations, with third form appeared at the beginning of stationary phase.

Specific metabolically-morphological pattern of red yeast C.capitatum was observed in this work. During start of stationary phase on agar plenty of micellar form appear and then the carotenoids was stored in membranes, while in liquid media (in fermentor) carotenoids were accumulated preferentially into lipid bodies and no significant signal from cytoplasmic membrane was detected up to $48^{\text {th }}$ hour. However, detailed investigation of this hypothesis is a matter of further research and comparison of various metabolic indicators.

\section{Acknowledgements}

The work was supported by the projects "Lipofungi“ - No. 268305 of the Research Council of Norway and "Materials Research Centre - Sustainability and Development" No. LO1212 of the Ministry of Education, Youth and Sports of Czech Republic.

\section{Conflict of interest statement}

The authors declare that they have no conflict of interest.

\section{References}

1. Irimia-Vladu M, Głowacki E, Voss G, Bauer S, Sariciftci N. Green and biodegradable electronics. Mater Today 2012; 15:340-346.

2. Irimia-Vladu M, Troshin P, Reisinger M, et al. Environmentaly sustainable organic field effect transistors. Organic Electronics 2010; 11:1974-1990.

3. Marova I, Carnecka M, Halienova A, Breierova E, Koci R. Production of Carotenoid-/Ergosterol-Supplemented Biomass by Red Yeast Rhodotorula glutinis Grown Under External Stress. Food Technol Biotechnol 2010; 48:56-61.

4. Frengova G, Beshkova D. Carotenoids from Rhodotorula and
Phaffia: yeasts of biotechnological importance. J Ind Microbiol Biotechnol 2009; 36:163-180.

5. Kandori H, Sasabe H, Mimurol M. Direct Determination of a Lifetime of the S2 State of Beta-Carotene by Femtosecond Time-Resolved Fluorescence Spectroscopy. J Am Chem Soc 1994; 116:2671-2672.

6. Xia S, Tan C, Zhang Y, Abbas S, Feng B, Zhang X, Qin F. Modulating effect of lipid bilayer-carotenoid interactions on the property of liposome encapsulation. Colloids and Surfaces B: Biointerfaces 2015; 128:172-180.

7. Schlee C, Miedl M, Leiper K, Stewart G. The Potential of Confocal Imaging for Measuring Physiological Changes in Brewer's Yeast. J Inst Brew 2006; 112:134-147

8. Murphy D. The biogenesis and functions of lipid bodies in animals, plants and microorganisms. Prog Lipid Res 2001; 40:325-438.

9. Murphy S, Martin S, Parton R. Lipid droplet-organelle interactions; sharing the fats. Biochimica et Biophysica Acta (BBA) - Molecular and Cell Biology of Lipids 2009; 1791:441-447.

10. Fujimoto T, Ohsaki Y, Cheng J, Suzuki M, Shinohara Y. Lipid droplets: a classic organelle with new outfits. Histochem Cell Biol 2008; 130:263-279.

11. Radulovic M, Knittelfelder O, Cristobal-Sarramian A, Kolb D, Wolinski $\mathrm{H}$, Kohlwein $\mathrm{S}$. The emergence of lipid droplets in yeast: current status and experimental approaches. Curr Genet 2013; 59:231-242.

12. Fei W, Shiu G, Zhang Y, Krahmer N, Ferguson C. A Role for Phosphatidic Acid in the Formation of "Supersized" Lipid Droplets. PLoS Genet 2011; 7:e1002201.

13. Rani S, Saha S, Rajasekharan R. A soluble diacylglycerol acyltransferase is involved in triacylglycerol biosynthesis in the oleaginous yeast Rhodotorula glutinis. Microbiology 2012; 159:155-166.

14. An G.H, Suh O.S, Kwon H.C, Kim K, Johnson E.A. Quantification of carotenoids in cells of Phaffia rhodozyma by autofluorescence. Biotechnol Lett 2000; 22:1031-1034.

15. Petrik S, Marova I, Haronikova A, Kostovova I, Breierova E. Production of biomass, carotenoid and other lipid metabolites by several red yeast strains cultivated on waste glycerol from biofuel production - a comparative screening study. Ann Microbiol 2013; 63:1537-1551.

16. Schneckenburger $\mathrm{H}$. Fluorescence decay kinetics and imaging of $\mathrm{NAD}(\mathrm{P}) \mathrm{H}$ and flavins as metabolic indicators. Opt Eng 1992; 31:1447-1451.

17. Fabregas J, Dominguez A, Maseda A, Otero A.: Interactions between irradiance and nutrient availability during astaxanthin accumulation and degradation in Haematococcus pluvialis. Appl Microbiol Biotechnol 2003; 61:545-551.

18. Cutzu R, Clemente A, Reis A, Nobre B, Mannazzu I, Roseiro J, Lopes da Silva T.: Assessment of $\beta$-carotene content, cell physiology and morphology of the yellow yeast Rhodotorula glutinis mutant 400A15 using flow cytometry. J Ind Microbiol 2013; 40:865-875.

19. Freitas C, Nobre B, Gouveia L, Roseiro J, Reis A, Lopes da Silva T.: New at-line flow cytometric protocols for determining carotenoid content and cell viability during Rhodosporidium toruloides NCYC 921 batch growth. Process Biochem 2014; 49: 554-562.

20. Freitas C, Parreira T, Roseiro J, Reis A, Lopes da Silva T.: Selecting low-cost carbon sources for carotenoid and lipid production by the pink yeast Rhodosporidium toruloides NCYC 921 using flow cytometry. Bioresour Technolog 2014; 158:355-359. 